\title{
Stellar population properties for 2 million galaxies from SDSS DR14 and DEEP2 DR4 from full spectral fitting
}

\author{
Johan Comparat* \\ Max-Planck-Institut für extraterrestrische Physik(MPE), Giessenbachstrasse 1, D-85748 \\ Garching bei München, Germany \\ E-mail: comparat@mpe.mpg.de
}

\begin{abstract}
We determine the stellar population properties - age, metallicity, dust reddening, stellar mass and the star formation history - for all spectra classified as galaxies that were published by the Sloan Digital Sky Survey (SDSS data release 14) and by the DEEP2 (data release 4) galaxy surveys [Abolfathi et al.(2017), Newman et al. (2013)]. We perform full spectral fitting on individual spectra, making use of high spectral resolution stellar population models [Maraston \& Strömbäck (2011), Wilkinson et al. (2017)]. Calculations are carried out for several choices of the model input, including three stellar initial mass functions and three input stellar libraries to the models. In total we provide up to nine modelling of the continuum for each galaxy. We perform spectral model fitting for each input M11 model: M11-ELODIE, M11-MILES and M11-STELIB [Le Borgne et al. 2003, Sánchez-Blázquez, P. et al. 2006., Falcón-Barroso et al. 2011, Prugniel et al. 2007] and for three choices of the stellar initial mass function (IMF), namely Salpeter, [Salpeter et al. 1955], Chabrier, [Chabrier 2003] and Kroupa, [Kroupa 2001]. Fig 1 shows an example of the firefly's outputs for a low redshift galaxy. We study the accuracy of parameter derivation, in particular the stellar mass, as a function of the signal-to-noise of the galaxy spectra. We find that signal to noise ratio per pixel around 20 (5) allow a statistical accuracy on $\log _{10}\left(M^{*} / M_{\odot}\right)$ of $0.2(0.4)$ dex, for the Chabrier IMF. For the first time, we study DEEP2 galaxies selected by their [OII] luminosity in the redshift range $0.83<z<1.03$ [Comparat et al. 2006]. We find that they have stellar masses with a flat number density in the range $10^{9}<M / M_{\odot}<10^{11.5}$. We publish all catalogs of properties as well as model spectra of the continuum for these galaxies in [Comparat et al. (2017)] and on the web, here https://firefly.mpe.mpg.de/v1_1_0/. This catalog is about twice as large as its predecessors (DR12) and will aid a variety of studies on galaxy evolution and cosmology.
\end{abstract}

Frontier Research in Astrophysics - III (FRAPWS2018)

28 May - 2 June 2018

Mondello (Palermo), Italy

${ }^{*}$ Speaker. 


\section{References}

[Abolfathi et al.(2017)] SDSS Collaboration, Abolfathi, Aguado et al. 2017. ArXiv e-prints 1707.09322

[Chabrier 2003] Chabrier. Galactic Stellar and Substellar Initial Mass Function. PASP Jul 2003

[Comparat et al. (2017)] Comparat, Maraston et al. 2017. Stellar population properties for 2 million galaxies from SDSS DR14 and DEEP2 DR4 from full spectral fitting. ArXiv 1711.06575

[Comparat et al. 2006] The evolution of the [O II], $\mathrm{H} \beta$ and [O III] emission line luminosity functions over the last nine billions year. MNRAS sep 20164611076.

[Falcón-Barroso et al. 2011] Falcón-Barroso et al. 2011. An updated MILES stellar library and stellar population models. AAP aug 2011532 A95

[Kroupa 2001] Kroupa. On the variation of the initial mass function. MNRAS 2001 Apr. 322231.

[Le Borgne et al. 2003] Le Borgne et al. 2003. STELIB: A library of stellar spectra at R 2000. AAP may 2003402433.

[Maraston \& Strömbäck (2011)] Maraston, \& Strömbäck, 2011, MNRAS, 418, 2785

[Newman et al. (2013)] Newman, Cooper, Davis, et al. 2013, ApJS, 208, 5

[Prugniel et al. 2007] New release of the ELODIE library: Version 3.1. 2007.

[Salpeter et al. 1955] Salpeter. The Luminosity Function and Stellar Evolution. ApJ 1955 jan 212161

[Sánchez-Blázquez, P. et al. 2006.] Sánchez-Blázquez, P. et al. 2006. Medium-resolution Isaac Newton Telescope library of empirical spectra. MNRAS sep 2006371703.

[Wilkinson et al. (2017)] Wilkinson, Maraston, Goddard, Thomas, et al. 2017, MNRAS, 472, 4297 
Figure 1: Example of a fit for a galaxy spectrum randomly taken from a SDSS plate, namely plate=0266, $\mathrm{mjd}=51602$, fiberid=4. This galaxy lies at redshift 0.127 . It has a $\mathrm{S} / \mathrm{N}$ around the 4000A break of 10.75 . The top panel shows the observed spectrum (grey line) and its modelling (coloured lines). Each model fit is characterized by the number of single-burst stellar population models (SSP) given in the caption. Gaps in the spectrum correspond to regions masked due to the presence of emission lines. The second panel shows the $\chi^{2}$ distribution per pixel for each model compared to a normal distribution (labeled $\mathrm{N}(0,1)$, dashed line). The third and fourth rows of panels show the derived galaxy parameters and their $1 \sigma$ uncertainties. On the third row from left to right: age vs. metallicity (Panel 1), age vs. stellar mass (Panel 2) and SSP weights vs. age of the individual SSP components (Panel 3). On the fourth row from left to right: Panel 1 shows mass vs. metallicity, Panel 2 shows mass vs. reddening and Panel 3 shows the SSP weights vs. metallicities of the individual SSP components. Results consistently point towards a super-solar metallicity of $10^{0.2} Z_{\odot}$, an old age of $10^{10} \mathrm{yr}$, an $\mathrm{E}(\mathrm{B}-\mathrm{V})$ between 0.15 and 0.2 . and a stellar mass about $10^{10.75}-10^{11} M_{\odot}$. STELIB-based models give the most massive and older solution than MILES and ELODIE. The decomposition in SSPs shows the solution is constituted of two old bursts, one with solar metallicity and one with higher metallicity in comparable proportions.

PLATE $=0266, \mathrm{MJD}=51602, \mathrm{FIBERID}=0004, \mathrm{z}=0.127$
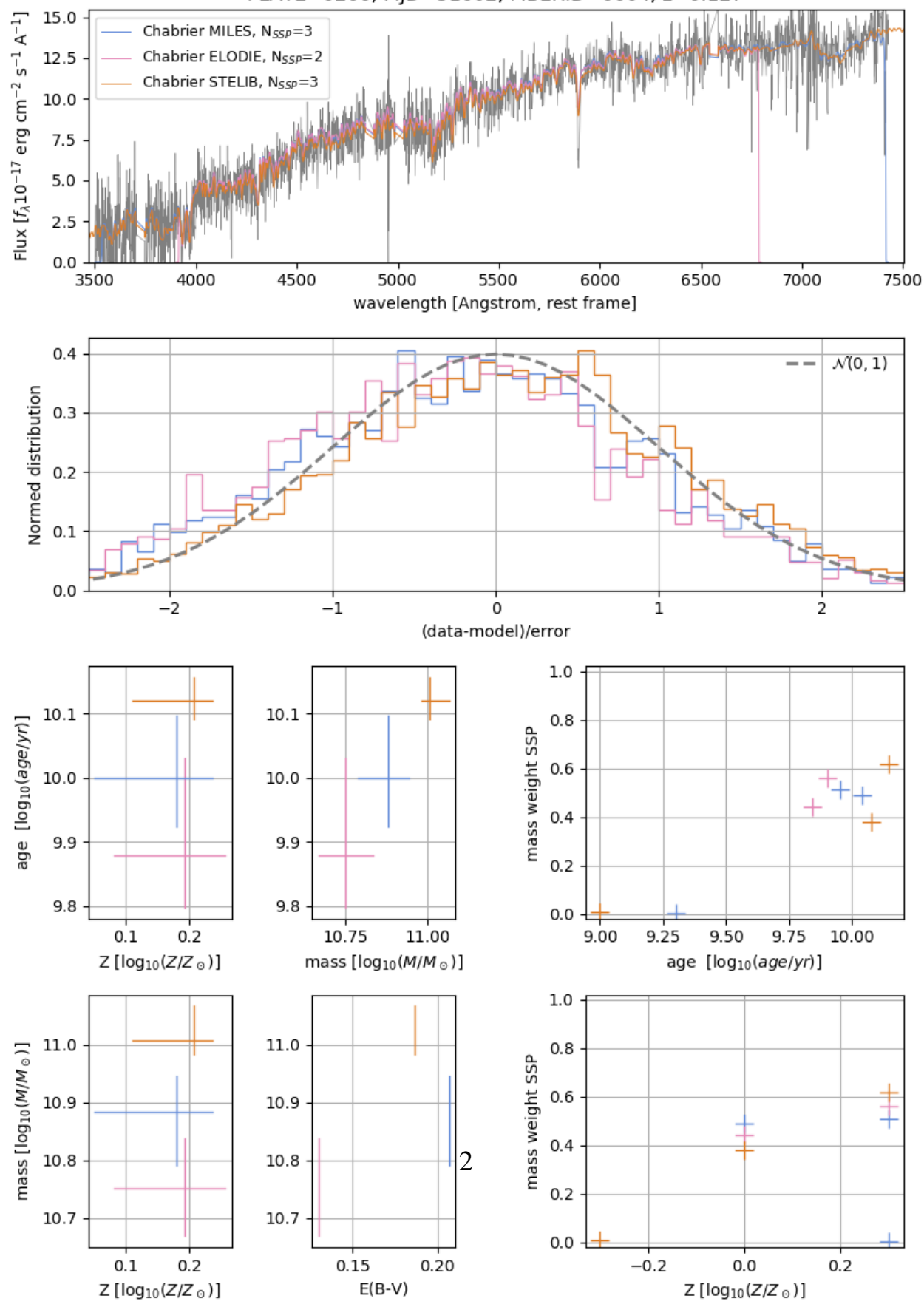